\title{
Repair of Sciatic Nerve Lesion Augmented with Amnion Membrane Composite and Mesenchymal Stem Cell
}

\author{
Heri Suroto $^{1}$ iD, R. Taufan Mulyo Wibisono ${ }^{1}$ \\ ${ }^{1}$ Department of Orthopaedic and Traumatology, Faculty of Medicine, Universitas Airlangga/ Dr. Soetomo General \\ Hospital \\ Surabaya - Indonesia
}

Correspondence should be addressed to Heri Suroto, Department of Orthopedic and Traumatology, Faculty of Medicine, Universitas Airlangga/ Dr. Soetomo General Hospital, J1. Mayjen Prof. Dr. Moestopo 6-8, Surabaya 60286, Indonesia. e-mail: heri-suroto@fk.unair.ac.id

\begin{abstract}
Background: Peripheral nerve injuries (PNI) are quite common, with an incidence of $13.9 \%$ per 100,000 people per year. The most cause is direct penetrating trauma. PNI will cause a significant decrease in the quality of life of patients.

Case: A 21-years-old woman complained of the inability to move her right leg and numbness. She had been injured in the of the right thigh due to broken glass. The patient underwent emergency surgery to repair the right Femoral artery and the Sciatic nerve.

Discussion: After six months since the first operation, flail foot and anesthesia were persisting with a FADI score of 47.1. We decided to perform re-exploration, from the exploration we found neuroma on the sciatic nerve. Excision was performed on the Neuroma then an end-to-end anastomosis was performed on both sciatic nerve components (tibial and peroneal) and augmented using amniotic membrane composites and mesenchymal stem cells. After 6 months since the second surgery, the patient could perform ankle dorsal flexion. After 12 months postoperatively, the patient could move his right leg functionally, sensory function also improved. Patients can undergo daily activities without difficulty, with a FADI score of 96.2.

Conclusion: There is a significant improvement in the patient's foot function after surgery using the method we used, more research is needed to optimize the use of amniotic membrane and mesenchymal stem cells as augmentation in nerve repair.
\end{abstract}

Keywords: Sciatic nerve lesion; Amniotic membrane; Mesenchymal stem cells; Human and medicine

\section{INTRODUCTION}

Peripheral nerve injury (PNI) is an injury that can have a significant effect on patient's lives. In Iran, PNI occurred in $1.3 \%$ of 16.753 cases of musculoskeletal trauma, laceration by a sharp object was the most common mechanism. ${ }^{1}$ Whereas in the US the prevalence of PNI is $1.64 \%$ from 220,593 cases of trauma with the most common cause was crush injuries. ${ }^{2}$
The more severe nerve damage, the longer the recovery period, and the more likely it is not to be able to recover. Suturing the injured nerve can be done primary or secondary, but if there is a major gap, it needs to be done by nerve grafting. ${ }^{3,4}$ Currently, the method used to improve repair of nerve injury that including nerve conduit, nerve cylinder, and augmentation by using mesenchymal stem cells (MSCs). The use of mesenchymal stem cells is to enable effective regeneration with the support of growth factors. 
Nerve conduit was introduced in 1984 which also can help nerve regeneration. The use of nerve conduits is as an alternative to autograft. Nerve conduits can be used in nerve injuries with gaps that reach $15 \mathrm{~mm} .{ }^{4}$

\section{CASE REPORT}

A 21-years-old woman complained unable to move her right foot and numbness. She had complained it for six months after she fell and the right thigh injured by broken glass, at the time she immediately underwent an emergency operation to repair the sciatic nerve and the right femoral artery. The patient routinely underwent physiotherapy but after 6 months, the patient still unable to move her right leg and the sensory compromise remained.

From the physical examination of the lower extremity region, postoperative scars were obtained in the posterior femur, significant atrophy of the lower leg muscle, flail foot, anesthesia in anteroposterolateral of lower leg, dorsal and plantar of the right foot, negative Achilles reflexes. The patient cannot actively move her ankle joints in plantar flexion, dorsal flexion, inversion, and eversion, passive ankle range of motion equal to the contralateral side. Foot \& Ankle Disability Index (FADI) score was 47.1. Neurophysiology examination shows chronic denervation of all lower leg muscles.

\section{Surgery Technique}

Neuroma on the sciatic nerve was located as high as $15 \mathrm{~cm}$ proximal from the popliteal crease with a length of $2 \mathrm{~cm}$. Both Tibial and Peroneal divisions at proximal and distal from the neuroma were intact (Figure 1). After neuroma excision, the location of the lesions and stitches in the previous surgery can be identified (Figure 2 and 3). We performed external neurotization of the sciatic nerve, stump freshening then identified the proximal and distal stump fascicles (Figure 4). End-to-end suture on each of the fasciculus (Figure 5). Then both nerves division was wrapped with membrane amnion that had been seeded with mesenchymal stem cells originated from autologous adipose tissue (Figure 6 and 7).

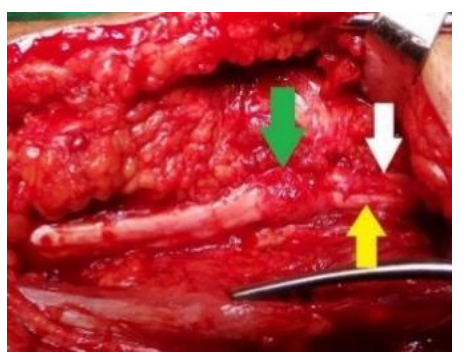

Figure 1. Neuroma on the right sciatic nerve (green arrow), tibial division (white arrow), peroneal division (yellow arrow).

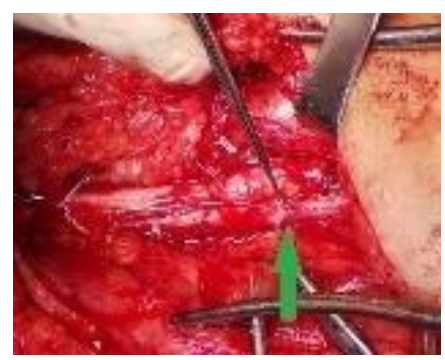

Figure 2. Location of lesions and sutures from previous surgery on the Peroneal division of the right sciatic nerve (green arrow).

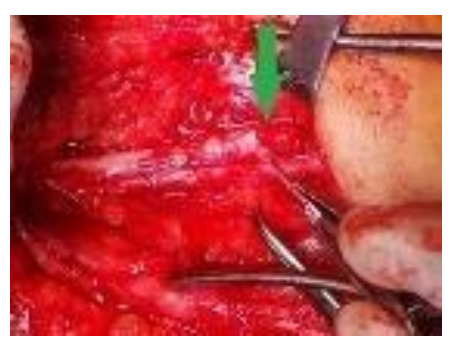

Figure 3. Location of lesions and sutures from previous surgery on the right Tibial division (green arrow). 


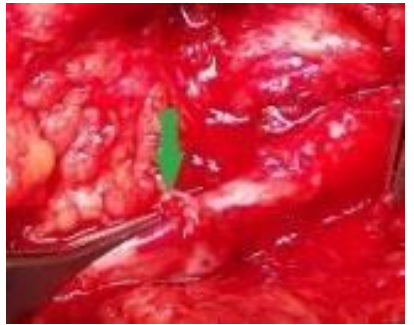

Figure 4. Fascicles in the proximal stump of the common peroneal division of the right Sciatic nerve.

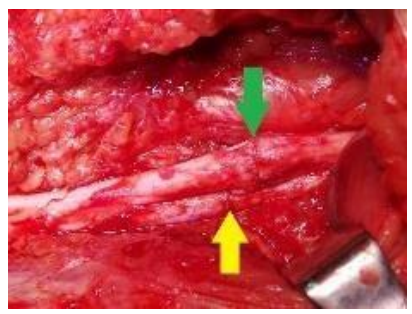

Figure 5. End-to-end fascicles sutures on the site of the lesion on both nerve division.

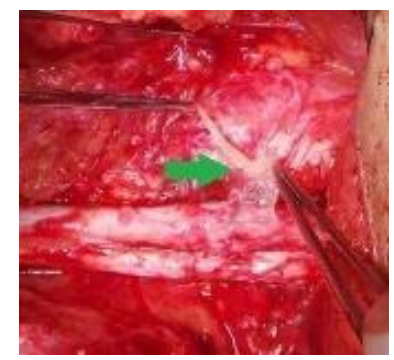

Figure 6. The wrapping process of the junction site with membrane amnion that has been seeded with MSC (green arrows)

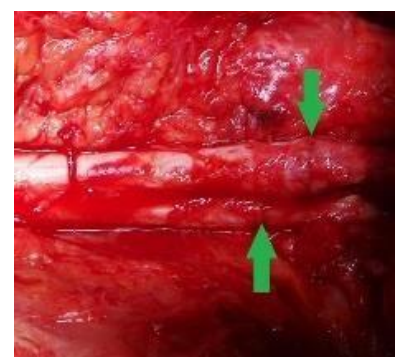

Figure 7. Junction site of Sciatic nerve lesion that has been wrapped in the amniotic membrane (green arrow).

\section{Post-Operative Evaluation}

At six months postoperatively, our patient was evaluated clinically and functionally using the FADI score. On physical examination, the surgical wound was healed, sensory function on the foot plantar area was improved, active movement in the ankle joint was $15^{\circ}$ of plantar flexion with motoric power of 3 (Figure $8 \mathrm{~A}$ ), $5^{0}$ of dorsal flexion with motoric power of 2 (Figure $8 \mathrm{~B}), 0^{0}$ of eversion with motoric power of $1,5^{0}$ of inversion with motoric power of 2 . The FADI score of 75 (FADI maximal score: 104).

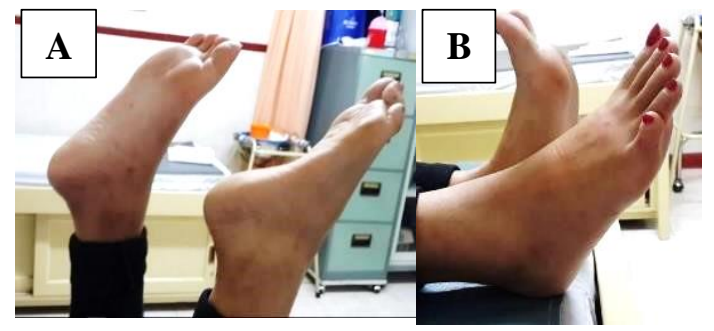

Figure 8. Plantar Flexion (A) and Dorsal Flexion (B) after six months postoperative

At 12 months postoperative, active movement at the ankle joint was $45^{\circ}$ of plantar flexion with motoric strength 5 (Figure 9A), $15^{0}$ of dorsal flexion with motoric strength 4 (Figure 9B), $15^{0}$ of inversion with motoric power of 4-, $10^{0}$ of eversion with motoric power 3 . The FADI score of 96.2 .

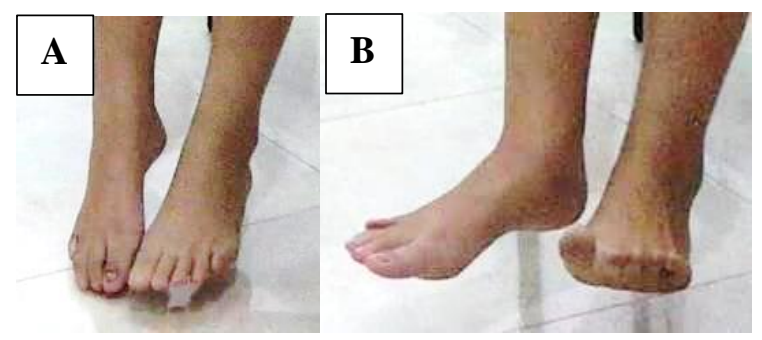

Figure 9. Plantar flexion (A) and dorsal flexion (B) 12 months postoperatively

\section{DISCUSSION}

Seddon divides peripheral nerve damage into three degrees: neuropraxia; axonotmesis; and neurotmesis. Neurotmesis occurs in this patient where there is an interruption of continuity of all nerve elements, so the risk of permanent nerve damage is very high because in neurotmesis there will be Wallerian degeneration followed by endoneurial tube damage accompanied by 
fibrosis that will prevent the regeneration of axons from entering the distal segment to reach the target segment organ. ${ }^{4,5}$

Regeneration of axons from the proximal stump will pass through the critical zone to arrive at the distal stump. Successful of regeneration, depending on what will happen at this level. When nerve fibers ruptured, nerve endings will be left with a particular gap. The zone between stumps shows the characteristics of exudate, cell proliferation, and collagen synthesis. At the initial stage, the gap will be filled with a lot of exudates containing corpuscles of blood and macrophages, and then fibrin will be formed. Furthermore, capillary and fibroblast will arise from nerve stumps along with those around it. Schwann cells move to the gap region of the distal and proximal stumps, forming a column and axon group. The structure of the medium affects the direction of the growing axon. ${ }^{6}$

Primary nerve suturing can be done after nerve transection. Primary suturing has an advantage, where the nerve endings have not yet been drawn far, and fibrosis tissue has not formed. In clean-cut nerve injuries can be made directly by using a fine thread but there should be no tension in the suturing. ${ }^{7}$

In our patient, the primary nerve suturing is performed during an emergency surgery, unfortunately after 6 months later there is no clinical sign of sciatic nerve reinnervation even though the patient has been undergoing a routine physiotherapy program, this is likely to occur because the fascicles of the distal and proximal stump were not on the correct position when sutured. Therefore, we did stumps freshening, meticulously identification, and sutured the fascicles precisely.

Our goal of using amnion membrane composite and mesenchymal stem cells in our surgery is to accelerate and optimize the regeneration process. In previous studies, the use of MSC to regenerate nerve showed a good result. ${ }^{8,9}$ We use the amnion membrane composite as a scaffold of MSC. The amnion membrane contains many growth factors, so we assume that the amnion membrane will stimulate proliferation and differentiation of MSC which will optimize and accelerate the healing of nervous tissue. ${ }^{10}$

We evaluate clinical improvement at 6 and 12 months postoperatively using the FADI score. The initial FADI score was 47.1; 6 months post-operatively was 75 ; and 12 months postoperatively was 96.2 and the patient does not experience significant difficulties in her daily activities.

\section{CONCLUSION}

Evaluation of clinical conditions in this patient shows good functional results. Further research is needed regarding the use of amnion membrane composite and MSC as augmentation in the repair of a nerve lesion.

\section{REFERENCES}

1. Saadat S, Eslami V, Rahimi-Movaghar V. The incidence of peripheral nerve injury in trauma patients in Iran. Ulus. Travma ve Acil Cerrahi Derg. 2011; 17(6): 539-44.

2. Taylor CA, Braza D, Rice JB, Dillingham $\mathrm{T}$. The incidence of peripheral nerve injury in extremity trauma. Am J Phys Med Rehabil. 2008; 87(5): 381-5. 
3. Asplund M, Nilsson M, Jacobsson A, Von Holst $\mathrm{H}$. Incidence of traumatic peripheral nerve injuries and amputations in Sweden between 1998 and 2006. Neuroepidemiology. 2009; 32(3): 217-28.

4. Ramachandran S, Midha R. Recent advances in nerve repair. Neurol India. 2019; 67(7): 106.

5. Solomon L, Warwick D, Nayagam S. Apley's System of Orthopaedics and Fractures. Ninth Edition. London: Hodder Arnold: An Hachette UK Company; 2010.

6. Griffin M, Malahias M, Hindocha S, Khan WS. Peripheral Nerve Injury: Principles for Repair and Regeneration. Open J. Orthop. 2014; 8(1): 199-203.

7. Moore AM, Wagner IJ, Fox IK. Principles of nerve repair in complex wounds of the upper extremity. Semin Plast Surg. 2015;
29(1): 40-7.

8. Masgutov R, Masgutova G, Mullakhmetova A, Zhuravleva M, Shulman A, Rogozhin A, et al. Adiposederived mesenchymal stem cells applied in fibrin glue stimulate peripheral nerve regeneration. Front Med. 2019; 6 (68): 112.

9. Cooney DS, Wimmers EG, Ibrahim Z, Grahammer J, Christensen JM, Brat GA, et al. Mesenchymal Stem Cells Enhance Nerve Regeneration in a Rat Sciatic Nerve Repair and Hindlimb Transplant Model. Sci Rep. 2016; 6:1-12.

10. Mamede AC, Carvalho MJ, Abrantes AM, Laranjo M, Maia CJ, Botelho MF. Amniotic membrane: From structure and functions to clinical applications. Cell Tissue Res. 2012; 349(2): 447-58. 